\title{
VOLUME 62
}

INDEX OF SUBJECTS AND AUTHORS

Only main topics are listed, and reference is given to the first page of each paper in which they are discussed

Adler, I. D., 139

Ageing, Sex and DNA repair. C. Bernstein and $\mathrm{H}$. Bernstein - book review, 79

Aguadé, M., 101

Anderson, W. W., 63

Archibald Garrod and the Individuality of Man. A. G. Bearn - book review, 163

Audio Cassettes of the 17th International Congress of Geneticsbook review, 243

bacteriophage T4, Luria-Latarjet effect, I

Balter, H., 111

Barton, N. H., 77, 149

Bellec, C., 89

Berrebi, P., 89

Biology of Mosquitoes, Vol. I: Development, Nutrition and Reproduction. A. N. Clements - book review, 82

Birchler, J. A., 15

Bishir, J., 57

book reviews, $77,159,243$

books received, $87,167,245$

Bownes, M., 82

Brassica juncea chloroplasts, 11

Brock, D., 82, 161, 163, 164

Brookfield, J. F. Y, 23

Buck, C., 133

Bulfield, G., 195

Bulinus truncatus genetic variation, 89

Causes of Molecular Evolution. J. H. Gillespie - book review, 77 chloroplast genome recombination, 11

Chopra, V. L., 11

Comparative Method in Evolutionary Biology. P. H. Harvey and M. D. Pagel - book review, 81

Coturnix coturnix japonica, 45

Cyrtodiopsis dalmanni, sexual selection, 213

Cystic Fibrosis: Current topics. Ed. J. A. Dodge et al.-book review, 161

Delay, B., 89

density-dependent selection, 89

Drosophila melanogaster,

$b b$ (bobbed), 205

P-M hybrid dysgenesis, 111

retrotransposons, 125

$w$ (white) linkage disequilibrium, 101

$w$ (white) modifiers, 15

Wolbachia symbiont, 23

$Y^{\text {to }}, 205$

Drosophila pseudoobscura, fertility selection, 63

Endow, S. A., 205

favoured alleles in subdivided population, 149

Gebhardt, M. D., 63

Gebhardt-Henrich, S. G., 45
Genetic Disorders and the Fetus: Diagnosis, Prevention and Treatment - book review, 82

Genetic Effects on ageing II. Ed. D. E. Harrison - book review, 79 Genome Analysis-Strategies for physical mapping, Vol. 4. Eds K. E. Davies and M. Tilghman - book review, 83

Glass, S. J., 205

Gray, A., 3

Haley, C. S., 177

Handbook of Quantitative Forest Genetics. Eds L. Fins et al. - book review, 164

Harris, A., 161

Hastings, I. M., 169, 177

Hill, W. G., 244

Holden, P. R., 23

Human Gene Mutation. D. N. Cooper and M. Krawczak - book review, 161

Hyman, P., 1

Introduction to Basic Cell Culture: Animal Cell Culture. S. J. Morgan and D.C. Darling - book review, 160

Jarne, P., 89

Jones, P., 23

Kaufman, M. H., 18

Keightley, P. D., 125

Keightley, P. D., 195

Kirti, P. B, 11

Knott, S. A., 177

Komma, D. J., 205

Langley, C. H., 101

Leach, D. R. F., 159

Lee, S. J., 164

Leigh Brown, A., 81

Levitan, M, 111

Lyme Disease. Molecular and Immunologic Approaches. Ed. S. E. Schutzer - book review, 160

Manipulation of the Avian Genome. Eds R. J. Etches and A. M. V. Gibbins - book review, 84

Margulies, L., 111

Marks, H. L., 45

mass selection and interbreeding, 231

Mechanisms of Molecular Evolution. Eds N. Takahata and A. G. Clark - book review, 244

Miyagawa, K., 160

Miyashita, N. T., 10

Mohapatra, T., 11

mouse,

digynic triploid, 183

fat selection, 169, 177

Hyp (hypophosphatemia), 39

oocytes, 183

ornithine decarboxylase, 3 I

$T c d-3$ (t complex distorter-3), 133 
weight selection, 169,177

$X$ chromosome, parental imprinting, 139

Mulants at zantha and albina Loci in relation to Chloroplas Biogenesis in Barley. K. W. Henningsen et al.-book review, 165

Namkoong, G., 57

Narasimhulu, S. B., 11

neutral theory, test of, 223

Njiokou, F., 89

Nuttail, P. A., 160

Nuzhdin, S. V., 125

Partridge, L., 79

Pasyukova, E. G., 125

Power of Bacterial Genetics: A Literature based course. J. Beckwith and T. R. Silhavy - book review, 243

Prakash, S., 11

Preimplantation Diagnosis of Genetic Diseases, Eds Y. Verlinsky and $A$. M. Kuliev - book review, 164

Qiu, Z. Q., 39

quantitative trait loci, 195

Reeve, E. C. R., 243

retrotransposons, 125

Roberds, J. H., 57
Sabl, J. F., 15

Scriver, C. R., 39

Short course in Bacterial Genetics. J. H. Miller - book review, 159 Silver, L. M., 133

Skibinski, D. O. F., 223

Stevens, L., 84

Tada, T., 139

Tait, A., 31

Takagi, N., 139

Tenenhouse, H. S., 39

Thompson, R., 231

Thomson, A., 162

van Heyningen, V., 83

Veerkamp, R. F., 169, 177

Wang, Y., 111

Ward, R. D., 223

Webb, S., 183

West, J. D., 183

Wilkinson, G. S., 213

Wolbachia symbiont, 23

Woodwark M., 223

Woolliams, J. A., 231

Wray, N. R., 231 